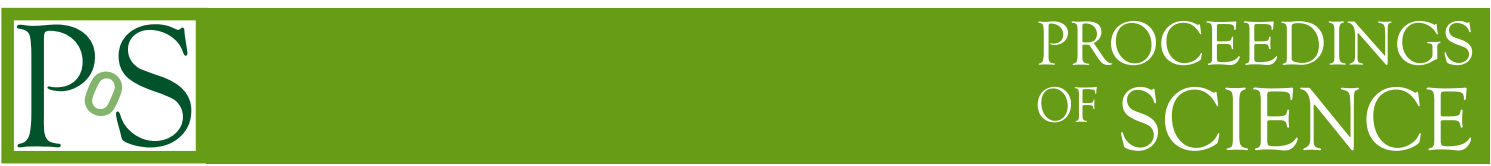

\title{
Searches for new physics with jets at ATLAS
}

\section{Lorraine Courneyea*†}

University of Victoria

E-mail: lorraine.courneyea@cern.ch

\begin{abstract}
Using data taken by the ATLAS detector at $\sqrt{s}=7 \mathrm{TeV}$, di-jet and multi-jet events were analyzed for evidence of new physics. The di-jet events were used to set a constraint on quark compositeness, while the multi-jets were used to probe for low-scale gravity and weakly-coupled string theory.

For the di-jet analysis, di-jet $\chi$ distributions were obtained from a dataset which corresponded to an integrated luminosity of $3.1 \mathrm{pb}^{-1}$. Analysis of the $\chi$ distributions excludes quark contact interactions at a $95 \%$ confidence level up to a compositeness scale of $3.4 \mathrm{TeV}$.

Through multi-body final states, a search was done for evidence of a fundamental scale of gravity, $M_{D}$, of order $1 \mathrm{TeV}$. Events with $\sum p_{T}>700 \mathrm{GeV}$ and invariant mass $>800 \mathrm{GeV}$ were selected from a sample with integrated luminosity of $(295 \pm 32) \mathrm{nb}^{-1}$. This yielded 193 events, all of which were multi-jet events. The number of events was in agreement with the QCD prediction of $254 \pm 18$ (stat.) \pm 83 (syst.), and an upper limit of $0.34 \mathrm{nb}$ at the $95 \%$ confidence level was set for new physics models with these final states.
\end{abstract}

Kruger 2010: Workshop on Discovery Physics at the LHC

December 5 - 10, 2010

Kruger National Park, Mpumalanga, South Africa

\footnotetext{
${ }^{*}$ Speaker.

${ }^{\dagger}$ On behalf of the ATLAS Collaboration
} 


\section{Introduction}

Various new physics models predict jets in their final states, making events with jets a potentially fruitful channel for searches at the LHC. Using data collected by the ATLAS detector at $\sqrt{s}=7 \mathrm{TeV}$, constraints were put on the quark compositeness scale and the cross section of interactions due to $\mathrm{TeV}$ scale gravity, two of the models which may be probed using jet final states.

\subsection{Quark Compositeness}

If quarks are composite objects, the effects of this would become visible above an energy scale $\Lambda$, known as the compositeness scale. If $\Lambda>\sqrt{s}$ interactions due to compositeness would be suppressed and the quarks would appear point like. The dominant effect would then be from a four fermion contact term in the Lagrangian. This term is:

$$
L_{q q q q}(\Lambda)=\frac{\xi g^{2}}{2 \Lambda_{q}^{2}} \bar{\Psi}_{q}^{L} \gamma^{\mu} \Psi_{q}^{L} \bar{\Psi}_{q}^{L} \gamma^{\mu} \Psi_{q}^{L}
$$

where $g^{2} / 4 \pi=1$. For the purpose of the di-jet analysis the choice of destructive interference with QCD was taken. The resulting difference in the exclusion limits between destructive $(\xi=1)$ and constructive $(\xi=-1)$ interference is on the order of $1 \%$ [1]. Note that this model may also be applied to other Beyond the Standard Model processes through an appropriate change in coupling.

To probe for evidence of contact interactions, the di-jet angular distributions are used. In particular, deviations from Standard Model QCD predictions of the di-jet angular distributions would be seen in the observable $\chi$, defined as:

$$
\chi=e^{\left|y_{1}-y_{2}\right|}
$$

where $y_{1(2)}$ are the rapidities of the two leading jets. Leading order QCD is dominated by t-channel gluon exchange which takes the form of Rutherford scattering, resulting in a mostly flat distribution in $\chi$ for QCD events. Contact interactions have a more isotropic angular distribution of final state jets, resulting in a distribution with a large enhancement at low $\chi$. Hence, the presence of new physics can be established through an excess of events at low $\chi$ as compared to the QCD prediction.

\subsection{TeV Gravity}

The motivation for $\mathrm{TeV}$ scale gravity stems from the hierarchy problem, which poses the question of why the electroweak scale, $M_{E W} \simeq 100 \mathrm{GeV}$, is so many orders of magnitude different than the Planck scale, $M_{P l} \simeq 10^{19} \mathrm{GeV}$. One way of addressing this problem is by postulating the existence of extra spatial dimensions through which only gravity propagates. An example of such models are ADD models [3] which postulate additional flat extra dimensions of length scale R. Gauss's law relates the D-dimensional fundamental scale of gravity, $M_{D}$, to the observed Plank scale in four dimensions by $M_{P l}^{2} \sim M_{D}^{n+2} R^{n}$. With appropriate choices of $\mathrm{R}$ and $\mathrm{D}$, this allows for the possibility that $M_{D}$ is on the order of $1 \mathrm{TeV}$.

A general signature of $\mathrm{TeV}$ gravity is multi-body final states. If $M_{D}$ is accessible at LHC energies, it becomes possible to create objects such as black holes and string balls with a large cross section. The decay of these objects via Hawking radiation leads to the multiplicity of particles in 
the final state. Under the assumption that gravity couples only to the energy-momentum content of matter, it is expected that the decays are democratic to all degrees of freedom in the Standard Model. This motivates the inclusion of all the low-mass Standard Model particles (electrons, photons, muons and jets) in the allowed final states. However, jets are expected to be the dominant final state objects, due to the greater number of degrees of freedom of quarks and gluons.

The observable of interest in the ATLAS TeV gravity search is the invariant mass, $M_{i n v}$, in high multiplicity events. The invariant mass is defined as:

$$
M_{i n v}=\sqrt{\left|p^{\mu} p_{\mu}\right|}
$$

where the four momentum $p^{\mu}$ is the sum of the four momenta of all the final state objects, plus a contribution from $E_{\mathrm{T}}^{\text {miss: }}$

$$
p^{\mu}=\sum_{i=\text { objects }} p_{i}^{\mu}+\left(E_{T}^{\text {miss }}, E_{x}^{\text {miss }}, E_{y}^{\text {miss }}, 0\right)
$$

To remain as general as possible, high multiplicity is defined as at least 3 objects with high $p_{T}$ $\left(p_{T}>20 \mathrm{GeV}\right.$ for photons, electrons and muons and $p_{T}>40 \mathrm{GeV}$ for jets) [2].

\section{Jet Reconstruction}

The anti-kt algorithm [4] was used to reconstruct jets in the ATLAS detector [5], with a size parameter of 0.6 for the quark compositeness analysis and 0.4 for the $\mathrm{TeV}$ gravity analysis. The inputs to this algorithm were topological clusters (3-D noise suppressed clusters) [5] calibrated at the EM-scale. An $\eta$ and $p_{T}$ dependent correction derived from Monte Carlo simulation [6] was applied to the resultant jets to correct the jets on average to the hadronic scale.

\section{Event Selection}

To ensure good data quality, the events used had to pass a series of selection criteria. All events were recorded during a period with stable LHC beam and ATLAS conditions and passed a L1 (hardware based) jet trigger. As well, to reduce non-collision background events, it was required that there was at least one primary collision vertex with five or more tracks. The position of the vertex also had to satisfy $|z|<30(15) \mathrm{cm}$ for the composite quark (TeV gravity) analysis.

Further refinements were made for the composite quark analysis, the $p_{T}$ of the leading (subleading) jet was constrained to be greater than 60 (30) $\mathrm{GeV}$ with both jets satisfying $|\eta|<2.8$. In addition, any event containing a jet with $p_{T}>15 \mathrm{GeV}$ which did not meet standard jet quality requirements was removed.

Selection criteria to reduce physics backgrounds were also applied to both analyses. These will be discussed in the context of each analysis.

\section{Quark Compositeness Search}

Events were binned according to their invariant mass, $m_{j j}=\sqrt{\left(E^{j 1}+E^{j 2}\right)^{2}-\left(\vec{p}^{j 1}+\vec{p}^{j 2}\right)^{2}}$. The highest $m_{j j}$ bin $\left(m_{j j}>1200 \mathrm{GeV}\right)$ has the greatest sensitivity to contact interactions, while the lower bins may be used to test the QCD Monte Carlo predictions for the shape of $\chi$. 


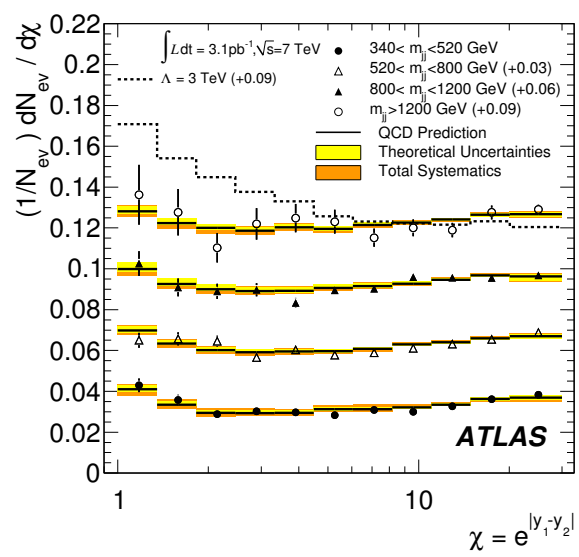

Figure 1: Normalized $\chi$ distributions for the various $m_{j j}$ bins, with plotting offsets shown in parentheses. Shown are the QCD predictions with systematic uncertainties, and data points with statistical uncertainties. The prediction for QCD with an added contact term with $\Lambda=3.0 \mathrm{TeV}$ is shown for the highest $m_{j j}$ bin.

Additional constraints were added to the selected events to ensure no artificial structure was added to the $\chi$ distributions. Defining $y *=\frac{1}{2}\left(y_{1}-y_{2}\right)$ and $y_{B}=\frac{1}{2}\left(y_{1}+y_{2}\right)$, where $y_{1(2)}$ is the rapidity of the first(second) jet, events are required to satisfy $y * \leq 1.7$ and $y_{B} \leq 0.75$. This results in events with $\chi<30$ and jets limited to $|\eta|<2.45$. In this region, acceptance is flat in $\chi$.

The resultant $\chi$ distributions found using $3.1 \mathrm{nb}^{-1}$ of data can be seen in figure 1 , along with a prediction for the case of a compositeness scale of $\Lambda=3.0 \mathrm{TeV}$. Good agreement is found with QCD, with a chi-square per degree of freedom of order 1 for all invariant mass bins.

An exclusion limit was set on $\Lambda$ by employing a frequentist analysis on the highest invariant mass bin $\left(m_{j j}>1200 \mathrm{GeV}\right)$. Defining $F_{\chi}$ as:

$$
F_{\chi}=\frac{N_{\text {events }}(\text { first } 4 \text { bins })}{N_{\text {events }}(\text { all bins })}
$$

where $0<\chi<3.2$ for the first four $\chi$ bins and $0<\chi<30$ for all the $\chi$ bins, 95\% confidence level intervals were established as a function of $\Lambda$. This was done by simulating several samples, each with a different value of $\Lambda$, then creating one sided $95 \%$ confidence level intervals for each sample, and interpolating between these results. Exclusion was taken to be where the $F_{\chi} 95 \%$ confidence level curve crossed the measured $F_{\chi}$ (figure 2). This resulted in a limit of $\Lambda \geq 3.4 \mathrm{TeV}$, corresponding to a distance scale of $6 \cdot 10^{-5} \mathrm{fm}$.

\section{TeV Gravity Search}

Previous limits constrain $M_{D}>800 \mathrm{GeV}$. Due to this, a search was done using events with $M_{i n v}>800 \mathrm{GeV}$. In addition, a restriction that events have $\sum p_{T}>700 \mathrm{GeV}$ was added in order to restrict the events to the central region and reduce contributions from QCD $2 \rightarrow 2$ scattering. These two criteria defined the signal region of the analysis. In order to establish the presence of a signal, a prediction for the number of QCD background events was necessary. To achieve this, the QCD Monte Carlo was normalized to the data in a control region, which consisted of events 


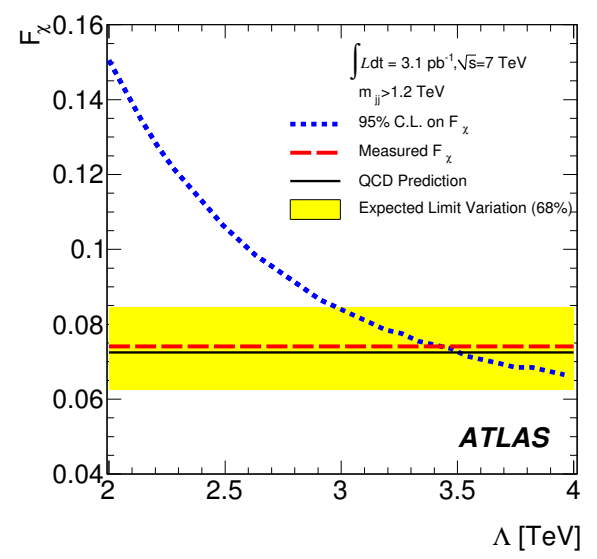

Figure 2: The dashed horizontal line is the measured $F_{\chi}$ and the solid horizontal line is the QCD prediction, with a band to illustrate a 1-sigma variation of the expected limit. The dotted curve is the 95\% CL exclusion contour for $F_{\chi}$ with quark contact interactions, used to set the exclusion limit on $\Lambda$.

with $300<M_{i n v}<800 \mathrm{GeV}$ and $\sum p_{T}>300 \mathrm{GeV}$. The selection of this region was based on the availability of statistics as well as the similarity of the kinematics to the signal region. The variation in the QCD background prediction due to the choice of control region was on the order of $10 \%$, and was taken as a systematic uncertainty.

For an integrated luminosity of $295 \pm 32 \mathrm{nb}^{-1}, 193$ events were found in the signal region. In these events, all the final state objects were jets. The Monte Carlo prediction for the number of QCD background events was $254 \pm 18$ (stat.) \pm 83 (syst.) events, in good agreement with the data. The data and Monte Carlo prediction in the signal region may be seen in figure 3 .

A limit was set on the acceptance times the cross-section of new physics due to $\mathrm{TeV}$ scale gravity using a Bayesian analysis and assuming a flat prior for the number of signal events. This gave an upper limit of $A \sigma=0.34 \mathrm{nb}$ at the $95 \%$ confidence level. Estimates of the acceptance were done using the BLACKMAX 2 [7] and CHARYBDIS 2 [8] event generators. Using BLACKMAX 2 an acceptance of 58\% was obtained, while the results from several samples generated by CHARYBDIS 2 were within $4 \%$ of this. This suggests an upper limit on the cross section of $0.6 \mathrm{nb}$, significantly lower than the cross-sections allowed by some theoretical models.

\section{Summary and conclusion}

Two limits on new physics were set using data collected by the ATLAS detector at $\sqrt{s}=7 \mathrm{TeV}$. The first limit was obtained using the $\chi$ distribution in di-jet events, and constrains the quark compositeness scale $\Lambda$ to be greater than $3.4 \mathrm{TeV}$, corresponding to a distance scale of $6 \cdot 10^{-5} \mathrm{fm}$. Previous results from D0 limited $\Lambda$ to be greater than 2.8 TeV[9], while the CMS collaboration has set a lower limit of $4.0 \mathrm{TeV}$ on $\Lambda[10]$. The second result is an upper limit on the cross section of new physics associated with $\mathrm{TeV}$ scale gravity. This was achieved through an analysis searching for an excess of events with multi-object final states and high invariant mass. The limit on the acceptance times the cross section for these processes was found to be $0.34 \mathrm{nb}$. 


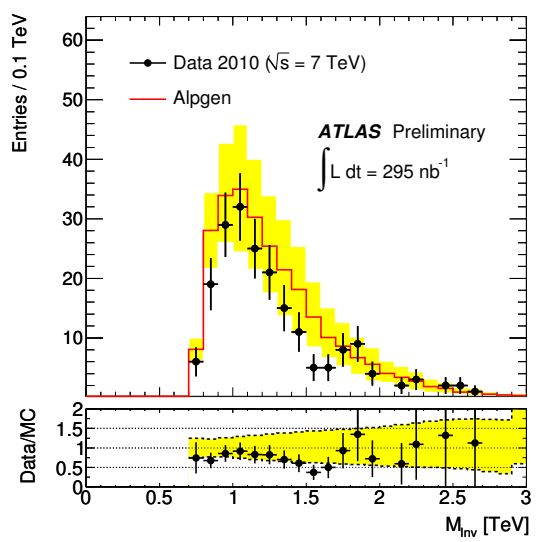

Figure 3: Invariant mass distributions for $\sum p_{T}>700 \mathrm{GeV}$. The solid black dots are the data, the red histogram is the ALPGEN background prediction, and the error band on the background is the total uncertainty.

\section{References}

[1] The ATLAS Collaboration, Search for Quark Contact Interactions in Dijet Angular Distributions in pp Collisions at sqrt(s) = 7 TeV Measured with the ATLAS Detector, Phys.Lett, B694 (2011) 327-345 [hep-ex/1009.5069]

[2] The ATLAS Collaboration, Search for new physics in multi-body final states at high invariant masses with ATLAS, Tech. Rep. ATLAS-CONF-2010-056,CERN, Geneva, Jul, 2010.

[3] N. Arkani-Hamed, S. Dimopoulos, and G.R. Dvali, The Hierarchy problem and new dimensions at a millimeter, Phys.Lett., B429 (1998) 263-272 [hep-ph/9803315]

[4] M. Cacciari, G.P. Salam, G. Soyez, The Anti-k(t) jet clustering algorithm, JHEP, 0804 (2008) 063 [hep-ph/0802.1189]

[5] The ATLAS Collaboration, The ATLAS Experiment at the CERN Large Hadron Collider, JINST 3 (2008) S08003.

[6] The ATLAS Collaboration Jet energy scale and its systematic uncertainty for jets produced in proton-proton collisions at sqrt(s) = $7 \mathrm{TeV}$ and measured with the ATLAS detector, Tech. Rep. ATLAS-CONF-2010-056, CERN, Geneva, Jul, 2010.

[7] D.C. Dai, G. Starkman, D. Stojkovic, C. Issever, E. Rizvi, et al., BlackMax: A black-hole event generator with rotation, recoil, split branes, and brane tension, Phys.Rev. D77 (2008) 076007 , [hep-ph/0711.3012]

[8] J.A. Frost, J.R. Gaunt, M.O. Sampaio, M. Casals, S.R. Dolan, et al., Phenomenology of Production and Decay of Spinning Extra-Dimensional Black Holes at Hadron Colliders, JHEP 0910 (2009) 014, [hep-ph/0904.0979]

[9] The D0 Collaboration Measurement of Dijet Angular Distributions at $\sqrt{s}=1.96 \mathrm{TeV}$ and Searches for Quark Compositeness and Extra Spatial Dimensions, Phys. Rev. Lett., 103, (2009) 191803, [hep-ex/0906.4819]

[10] The CMS Collaboration, Search for Quark Compositeness with the Dijet Centrality Ratio in pp Collisions at sqrt(s)=7 TeV, Phys. Rev. Lett. 105, (2010) 262001, [hep-ex/1010 . 4439] 